Musées, Patrimoine et Culture scientifiques et techniques

179 | 2018

septembre-octobre 2018

\title{
Libre de rire au muséum
}

Marie-Sylvie Poli, Caroline Archat et Ambre Mendoza

URL : http://journals.openedition.org/ocim/2753

DOI : $10.4000 /$ ocim. 2753

ISSN : 2108-646X

Éditeur

OCIM

Édition imprimée

Date de publication : 1 septembre 2018

Pagination : 20-27

ISSN : 0994-1908

Référence électronique

Marie-Sylvie Poli, Caroline Archat et Ambre Mendoza, "Libre de rire au muséum », La Lettre de l'OCIM

[En ligne], 179 | 2018, mis en ligne le 01 septembre 2019, consulté le 07 septembre 2019. URL : http:// journals.openedition.org/ocim/2753; DOI : 10.4000/ocim.2753

Ce document a été généré automatiquement le 7 septembre 2019

Tous droits réservés 


\title{
Libre de rire au muséum
}

\author{
Marie-Sylvie Poli, Caroline Archat et Ambre Mendoza
}

Manger, la mécanique du ventre : une exposition qui mêle sérieux scientifique et humour comme ce faux strip-tease.

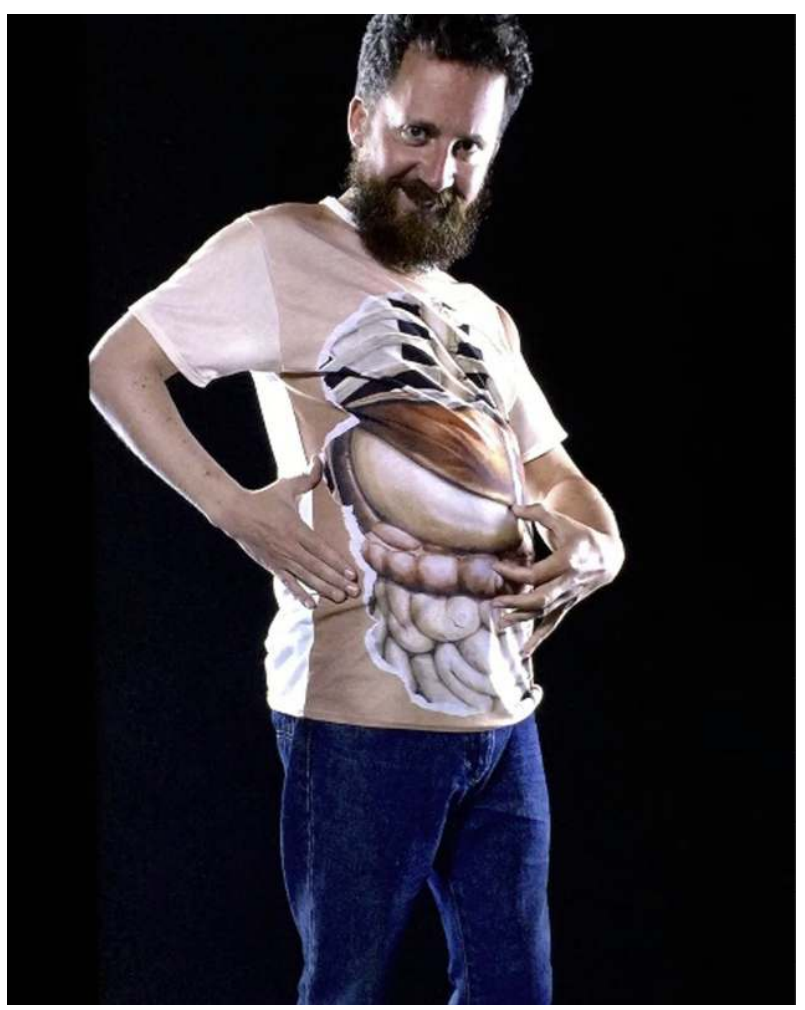

(C) Muséum de Neuchâtel

\section{Introduction}

1 Qui dans la vie courante ne prend pas plaisir à rire ou à sourire d'un bon mot, d'un canular, d'une histoire drôle, d'une image amusante ou d'une scène grotesque ? Peu de 
gens, nous sommes d'accord. Alors pourquoi ne pas laisser l'humour entrer au musée et le rire des visiteurs retentir dans les expositions ? Cette question apparemment triviale est selon nous un objet de réflexion parfaitement digne d'interpeler les acteurs contemporains du champ muséal. Et pourtant force est de constater un cruel déficit de travaux académiques sur l'humour dans la littérature de muséologie francophone. Dans les musées, les anglo-saxons sont plus enclins que leurs collègues francophones à opter pour des scénographies et des écritures expographiques mâtinées d'humour ou franchement désopilantes. Pourtant certains musées francophones, des musées de science en particulier, tentent l'expérience ${ }^{1}$. En Suisse c'est le cas du muséum d'Histoire naturelle de Neuchâtel (MHN) bien connu depuis plus de vingt ans pour ses expositions scientifiques qui n'hésitent pas à jouer avec les palettes des émotions et de l'humour pour intéresser, interpeler et fidéliser ses visiteurs ${ }^{2}$. Le succès est-il pour autant au rendezvous ? Ce constat posé, il nous est apparu intéressant de répondre à la requête du MHN de mener une étude de réception qualitative de Manger, la mécanique du ventre ${ }^{3}$, une exposition scientifique conçue et présentée du 27 novembre 2016 au 26 novembre 2017. Comme chercheurs en culture et communication, nous envisageons toute étude de réception comme une recherche critique sur le rôle culturel du musée aujourd'hui, bien au-delà d'une rudimentaire enquête de satisfaction ponctuelle. Ainsi avons-nous pensé cette exposition temporaire thématique en relation dialectique avec le projet scientifique et culturel du MHN et les sujets scientifiques de société en vogue à cette période. Ce positionnement méthodologique implique qu'avant de questionner les visiteurs, nous avons interrogé les concepteurs de l'exposition sur leurs objectifs et sur leurs modalités de travail. Nous avons ensuite réalisé des observations des dispositifs humoristiques dans les salles d'exposition ainsi que quinze entretiens semi-directifs avec trente-quatre visiteurs venus seuls ou accompagnés, afin de percevoir les effets de cette écriture humoristique du point de vue de la compréhension du discours scientifique et du point de vue des types de relations qu'instaure cette écriture entre le MHN et ses publics ${ }^{4}$. 
À l'entrée de l'exposition, l'espace « Apériclub " propose un jeu pour entendre pets, rôts et mastication.
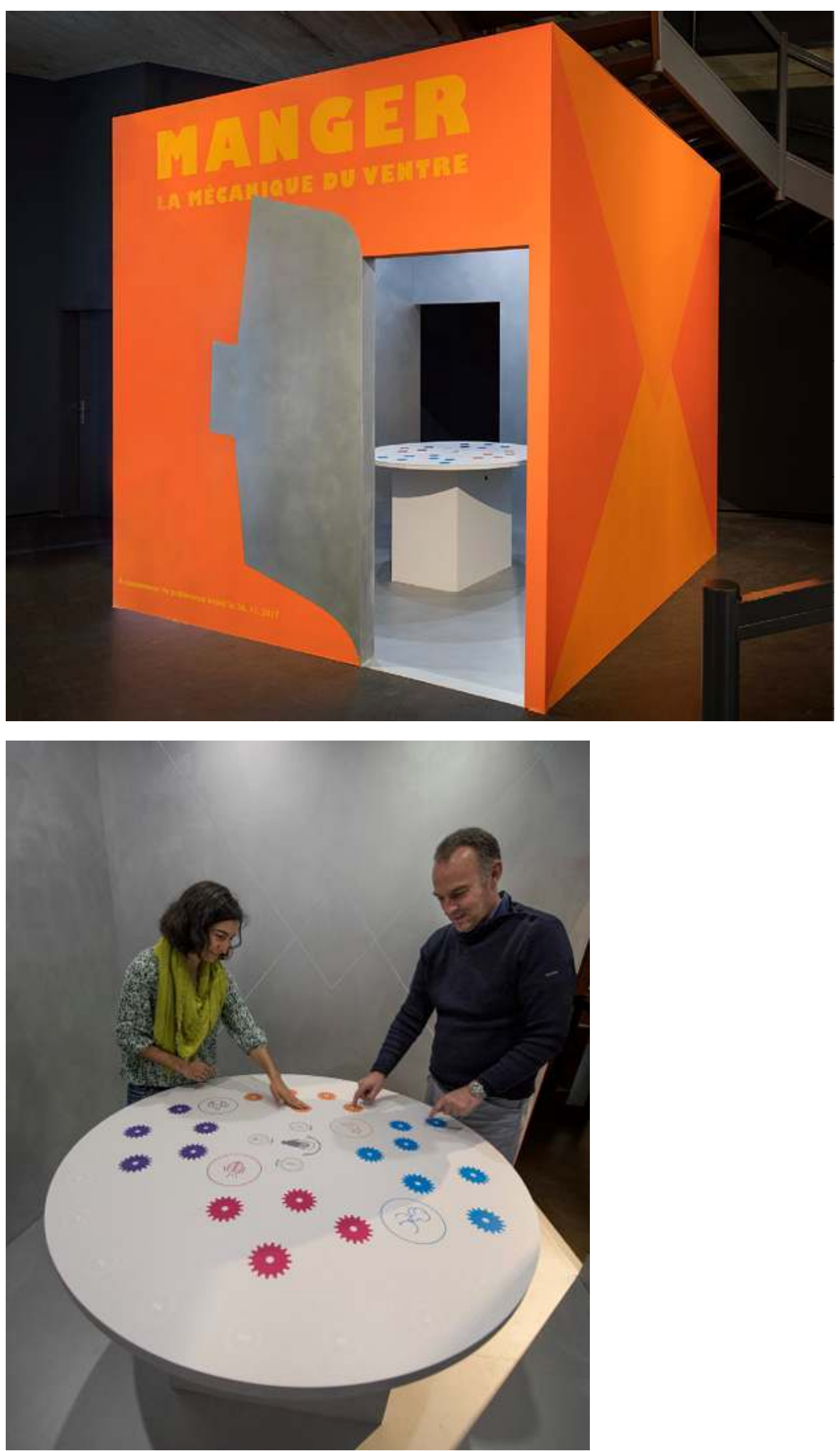

(c) Muséum de Neuchâtel

2 L'article débute par le rappel des objectifs visés par les concepteurs de l'exposition (commissaire, scénographe designer, médiateur, scientifique, artiste) et par indiquer 
l'intérêt de cette étude de réception pour le MHN. La seconde partie rend compte de nos résultats lorsque nous avons cherché à prendre le pouls du public en interrogeant les visiteurs sur la valeur, le rôle et l'intérêt qu'ils donnent à l'écriture humoristique dans Manger. En découle une troisième partie qui présente différentes figures de visiteurs, relatives à la variété des façons de vivre l'humour au musée.

\section{Un travail d'équipe dans lequel le recours à l'humour joue un rôle structurant}

Les entretiens avec les membres de l'équipe de conception de Manger mettent en évidence une méthode d'écriture collaborative aguerrie et rodée depuis des années.

Le chef d'orchestre est Christophe Dufour, directeur du MHN depuis trente six ans qui signait là sa dernière exposition avant que Ludovic Maggioni prenne la direction de l'établissement en octobre $2016^{5}$. Ces entretiens dévoilent un intense travail d'équipe polyvalente au quotidien, basé avant toute chose sur l'intérêt collectif pour des sujets scientifiques dont certains à dimension sociétale, sans injonction venue d'une tutelle politique ou scientifique, sans obligation de lien étroit avec les collections de l'établissement. À tous les entendre, on comprend que la polyvalence et la pluridisciplinarité gouvernent les rôles et les missions de création et de réalisation attribuées à chacune et à chacun. Autre trait de caractère de cette équipe, elle fait régulièrement appel à des créateurs ou à des artistes (comédiens, chanteurs, plasticiens, vidéastes, artistes numériques), leur laissant carte blanche pour leurs créations afin que chaque exposition ait son climat particulier, son ambiance unique. Le plaisir à faire découvrir des sujets scientifiques sans ennuyer et la transmission par l'humour avec une esthétique muséale inusitée reviennent sous les mots de chaque membre de l'équipe. Elles sont centrales, comme deux signatures revendiquées. Ce trait fédérateur autant conceptuel que pragmatique que nos entretiens mettent en lumière serait donc selon nous l'identité stylistique du MHN. Voyons comment, sur le terrain, l'humour et la chanson nourrissent l'exposition Manger. 


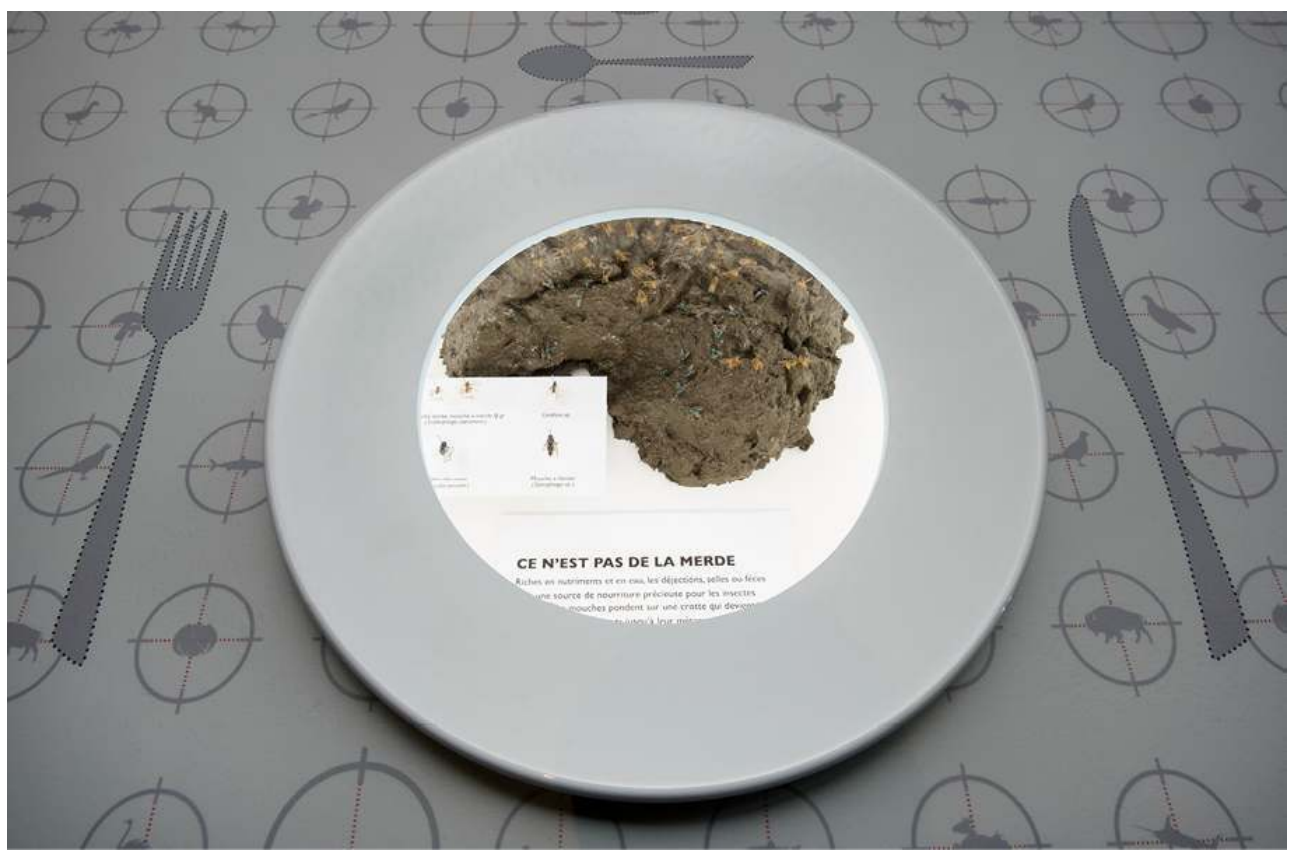

(c) Muséum de Neuchâtel

Manger, la mécanique du ventre qui aborde la question de l'alimentation dans le monde animal et humain est construite de façon linéaire en suivant le parcours des aliments dans le corps (de la bouche à l'anus). La démarche communicationnelle du musée repose sur le concept d'expositions-récits qui orientent la réception ${ }^{6}$. D'un point de vue scénographique, le parti pris a été de s'appuyer sur des éléments visuels pour créer le sentiment d'être au cœur du système digestif. Le visiteur entre dans le cœur de l'exposition par une vidéo représentant une grande bouche humaine qui mâche des aliments. Les références culturelles occupent une place importante dans le parcours. On y trouve l'évocation d'une nature morte et d'un banquet, célèbre conclusion des albums de la Bande dessinée Astérix. Une salle quasiment blanche est consacrée à l'explication des coutumes liées au jeûne humain et à la description de la physiologie des animaux qui jeûnent de façon régulière (l'anguille européenne, le manchot empereur, le tardigrade). Une salle où sont exposés des crottes en vitrine (humaines et animales), ainsi qu'un tirage photographique de l'anus étoilé d'une jument, provoque la surprise des visiteurs. Le parcours se termine par une salle dédiée aux implications de l'alimentation humaine sur l'environnement avec différents dispositifs numériques (tables interactives, quizz) dans un décor audacieux qui place le visiteur face à cinq figures comportementales. En structurant l'exposition de cette façon, le style du MHN fonctionne comme une signature d'auteur. Il y a là l'intention de présenter sur un même plan le monde animal et le monde humain et d'aborder crument la question de la défecation, phénomène que les convenances sociales ont tendance à dissimuler, voire à ignorer. Augmenté par l'omniprésence d'une scénographie théâtrale et immersive, le visiteur est saisi par un récit épique qui prend l'allure d'une aventure au cœur du vivant. 
Dans l'espace consacré à l'intestin, le visiteur doit se présenter au bureau de la douane où, en tant que glucide, protide ou graisse, il doit effectuer une déclaration pour franchir la paroi de l'intestin.

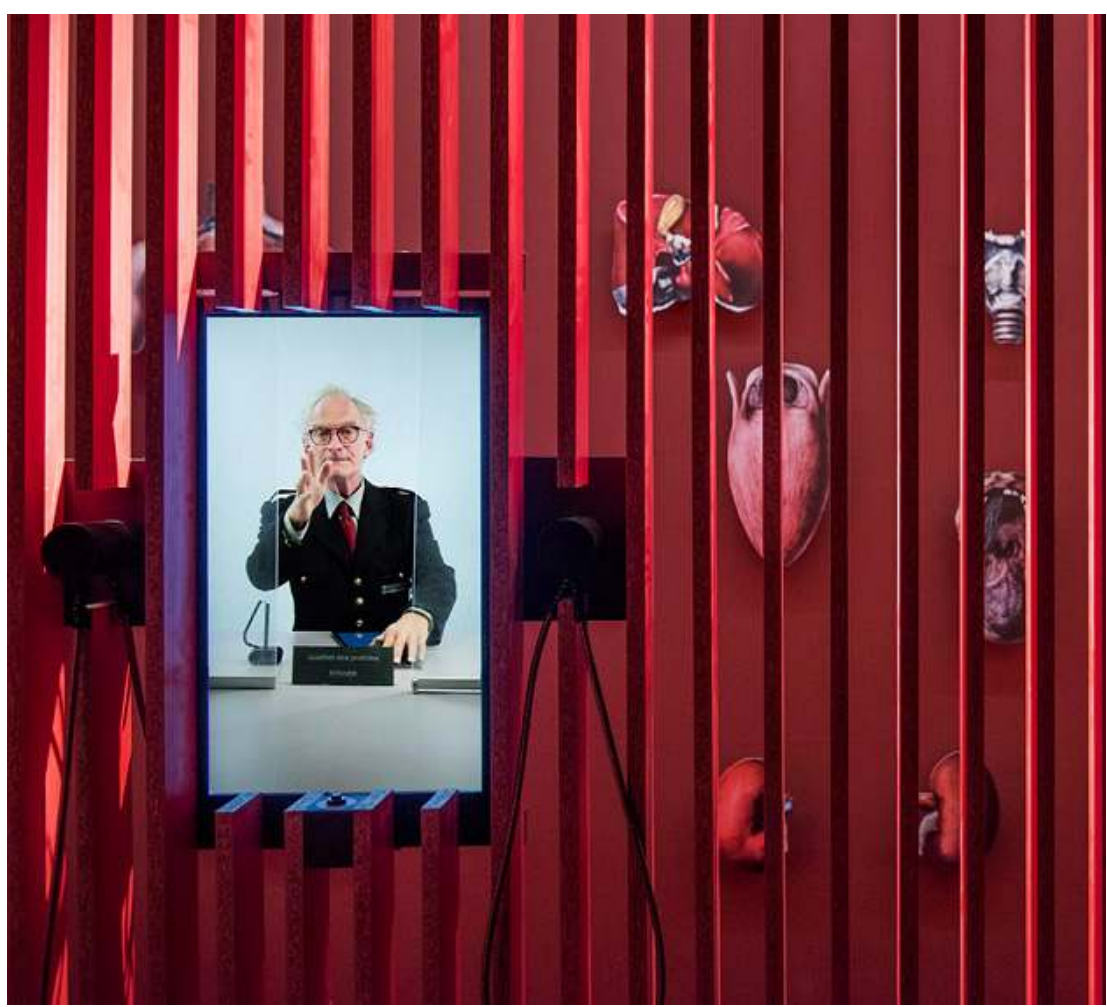

(c) Muséum de Neuchâtel

$6 \quad$ Tout au long du parcours, les informations scientifiques sont relayées par des dispositifs humoristiques sur des supports vidéo réalisés par des artistes. Les techniques numériques permettent ici de confier le discours à des personnages fictifs, décalés du monde scientifique, selon le procédé de «focalisation interne » tel que le conçoit Daniel Jacobi ${ }^{7}$. L'équipe du musée a sollicité le groupe Les Petits chanteurs à la gueule de bois, connu dans la région de Neuchâtel pour son répertoire de chansons grivoises ${ }^{8}$. Parmi les quatre chansons composées pour l'exposition, Hannibal est une complainte inspirée de la culture Hip-Hop qui raconte l'existence du ver solitaire. Les paroles de la chanson sont truffées de jeux de mots, de références aux récits de la mythologie grecque et de la Bible. La polysémie et la stylisation du discours (rimes et rythme binaire) installent une distanciation vis-à-vis de ce bothriocéphale que le sens commun considère comme repoussant. Le comique de langage servirait ici de levier communicationnel pour amuser, apprendre et tranquilliser. Autre exemple, la salle de l'intestin grêle est équipée de trois vidéos qui s'adressent directement aux visiteurs comme si ils se présentaient à un guichet de douane aéroportuaire de gastro-entérologie. Dans ce face à face, chaque visiteur change de posture, il devient un nutriment (glucide, protide ou graisse) qui cherche à passer à travers la paroi intestinale. L'acteur Philippe Vuilleumier, qui a déjà collaboré avec le MHN, joue ici le rôle du douanier gastroentérologue. Le dispositif tourne au canular, à la différence près que le visiteur est invité à se rendre complice de la comédie qui se joue. Sur le plan stylistique, les dispositifs humoristiques de l'exposition Manger mêlent plusieurs registres énonciatifs : le sérieux, le rigoureux d'une part ; le ludique, le comique, la dérision, l'insolite d'autre part. 
7 Pour autant, la validité scientifique des contenus est omniprésente, quels que soient les supports de communication. Ainsi le visiteur ne perdrait pas le fil du récit savant, même si, à plusieurs reprises, il est pris au jeu d'un divertissement non conventionnel, surpris par la façon de traiter la question. L'espace " Apériclub ", prélude à l'exposition, propose un jeu de table sonore dont les icônes peuvent être activées pour entendre des sons de pets, de rôts, de mastication. Les concepteurs présentent ici une vision décalée du thème qui, dès le début du parcours, évoque par la voix des Petits chanteurs à la gueule de bois le cycle de la vie et de la mort. L'humour mêlé des concepteurs et des artistes multiplie les possibilités d'aborder le thème de l'exposition selon des approches différentes qui se complètent. Le sérieux scientifique donne au divertissement une légitimité, et à l'inverse, la détente, les ritournelles ou la surprise d'un faux strip-tease en vidéo soutiennent l'attention. Les canulars en cascade conduisent le visiteur à une distanciation involontaire qui a le double privilège de nourrir une prise de conscience du phénomène digestif et de dédramatiser l'aspect organique du corps humain.

\section{Des visiteurs charmés et critiques à la fois}

8 Si l'équipe du musée postule que les dispositifs humoristiques de Manger divertissent et séduisent les visiteurs, allègent le propos scientifique et permettent d'entretenir une distance critique par rapport au sujet, qu'en est-il du retour des visiteurs eux-mêmes ? Les subtilités, les discordances de sens, le comique de langage ou de situation portés par la scénographie, la présence des chanteurs et du comédien sont-ils compris et appréciés par les visiteurs interrogés? Quelles sont les différentes façons d'appréhender l'humour dans l'exposition selon que l'on est un habitué du musée ou un visiteur de passage, un enfant ou un adulte, selon l'intérêt que l'on cultive vis-à-vis des sciences et des questions de société ou bien le sens de l'humour que l'on a développé ? En quoi l'humour dans cette exposition est-il un moyen de développer une plus grande conscience des enjeux de l'alimentation? Comme toute construction sociale, l'humour requiert un certain nombre de connaissances et d'aptitudes préalables. Le second degré, le décalage, la dérision ne sont pas accessibles si on ne connaît pas le point à partir duquel le déplacement de sens s'opère. On peut donc présumer différentes façons d'accéder aux significations de l'exposition selon l'âge des visiteurs, même si la pluralité des registres de communication permet des niveaux d'adresses multiples.

9 Les quinze entretiens réalisés avec trente-quatre visiteurs ${ }^{9}$ révèlent une grande richesse de contenus, une exigence soutenue vis-à-vis du musée, une importante capacité d'autodérision ainsi qu'une réflexion sincère sur l'expérience de visite. Il n'est pas possible ici de rendre compte de ce travail d'enquête de manière exhaustive. La sélection ci-après désigne les résultats les plus révélateurs des effets de l'humour dans le processus de découverte de l'exposition. Les dispositifs humoristiques (quatre chansons des Petits chanteurs à la gueule de bois, trois vidéos pour la comédie du douanier, une vidéo d'un strip-tease, une table sonore, etc.) sont fortement appréciés pour leur caractère comique et décalé. Les visiteurs découvrent une exposition « impressionnante », « inattendue » où il n'est pas nécessaire d'être toujours sérieux pour apprendre. Ils reconnaissent ainsi dans la démarche du MHN l'intention de leur offrir un moment agréable sans que l'exigence scientifique ne soit négligée. Nos analyses font apparaître également que l'écriture humoristique de l'exposition donne une sensation différente du temps effectivement passé dans le musée. Pour la majorité des visiteurs, elle rend la visite «plus digeste » et 
fait disparaitre l'ennui. Les mélodies des Petits chanteurs à la gueule de bois, au-delà d'être drôles, loufoques même, favorisent la mémorisation des contenus scientifiques. Certains signalent le rôle mnémotechnique de la chanson. Associés au divertissement et même à l'exubérance, les textes chantés déclenchent un discours désinhibé chez certains visiteurs qui reprennent les paroles des chansons et adoptent un ton humoristique au moment de l'entretien. Les trois vidéos du douanier, notamment celle qui évoque le passage des graisses dans l'organisme, provoquent un engouement chez les adultes. Ce succès repose aussi sur le fait que ces vidéos invitent le visiteur à quitter sa position de récepteur pour devenir l'acteur d'un canular. Tous ces dispositifs constituent une médiation vers les savoirs, au sens où ils tissent étroitement une expérience sensible et ludique avec des connaissances scientifiques. Cependant, si certains visiteurs déclarent lire les textes affichés autour des vidéos après les avoir regardées, nos observations attestent qu'ils sont peu nombreux. La plupart déclarent ne pas se sentir capables de tout lire. L'humour en chansons ou en vidéos remplacerait-il la lecture de textes au musée ? Répondre à cette question nécessiterait d'approfondir l'étude et de réaliser des observations systématiques sur une durée plus importante que celle dont nous avons disposé. Cela n'empêche pas de constater que les visiteurs que nous avons interrogés vivent une expérience particulière avec les savoirs. Dans un état d'esprit léger et drôle, ils n'échappent ni à une compréhension globale de la mécanique du ventre, ni à une clarification des similitudes et des différences entre le monde animal et le monde humain. Ils ne manquent pas non plus de se « rafraîchir la mémoire ", ni d'accéder à des connaissances complexes.

Certains savoirs acquis dans le parcours entraînent un réajustement des représentations : l'inutilité prétendue et maintenant contestée de l'appendice, la complexité de la digestion de la vache, ou encore la capacité de certains organismes à supporter l'absence de nourriture sur des durées importantes. L'exposition est donc plébiscitée pour le nombre et la qualité des connaissances qu'elle transmet. L'imbrication du plaisir, du divertissement, de la mémoire et des savoirs ressort également comme un moyen efficace pour contrer l'éventuel dégoût, l'inquiétude ou l'angoisse lié(é)s à la prise de conscience des implications environnementales de nos comportements alimentaires. Le pourcentage de personnes sous-alimentées est mémorisé parce qu'il interpelle la conscience du visiteur. La découverte de l'othorexie (obsession de manger des aliments considérés comme sains), considérée comme une maladie contemporaine, déclenche l'envie de "faire attention " à notre rapport à l'alimentation. Loin de se substituer aux propos scientifiques de l'exposition, l'humour qui soutient l'attention des visiteurs sur un sujet scientifique et sociétal semble ainsi consolider une complicité avec le musée. 
La bactérienne. Des bactéries animées entourent les chanteurs.

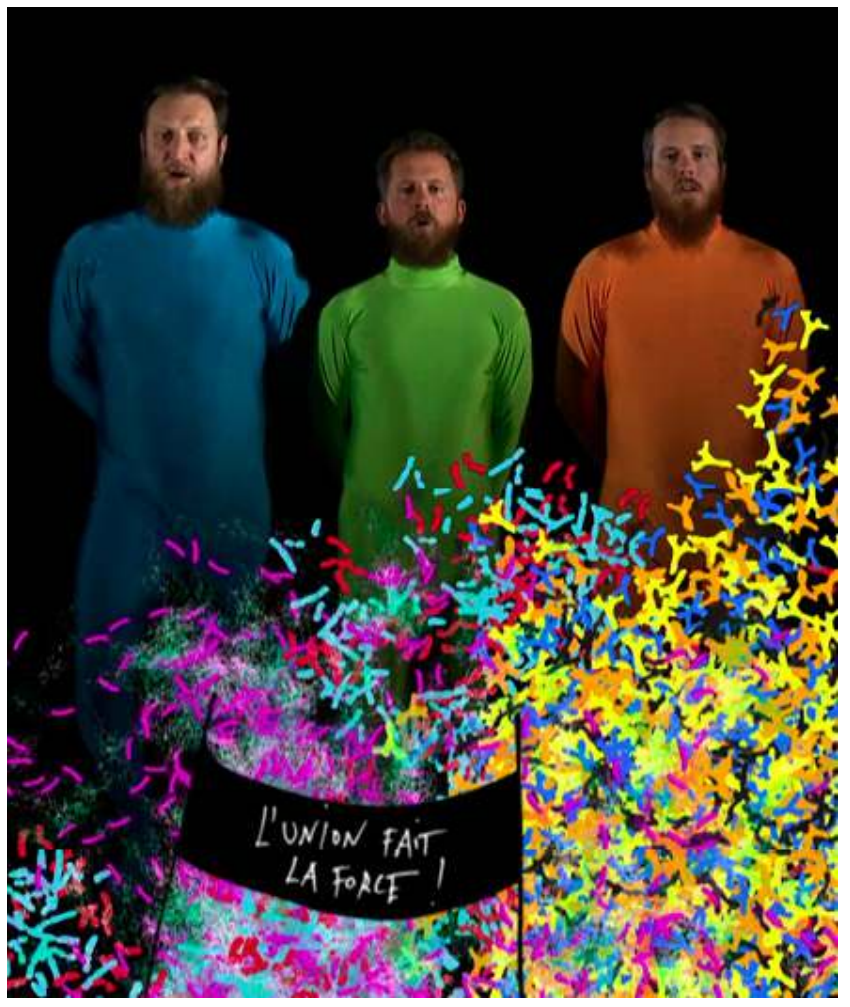

(c) Muséum de Neuchâtel

\section{Du côté des visiteurs, différentes manières de réagir à ce style de muséographie}

11 Nos analyses, partiellement restituées ci-dessus, nous permettent de dégager une typologie de figures de visiteurs de Manger. Ces figures ne sont pas des catégories étanches ou définitives. Elles sont plutôt des repères pour situer les différences d'expériences vécues. Nous avons ainsi dégagé la figure du « visiteur curieux » habitué du MHN qui porte un grand intérêt aux choix muséographiques du musée. En se questionnant sur la façon dont l'institution met en exposition un thème scientifique, il se place, en quelque sorte, du côté de l'équipe de conception avec qui il entretient une connivence. Le mobile de sa visite réside de façon primordiale dans l'observation des manières innovantes ou décalées de faire une exposition au MHN et dans le désir d'être surpris par une démarche originale. À l'opposé de cette posture, nous avons repéré la figure $d u$ «visiteur personnel» qui centre son attention sur la façon dont les connaissances exposées vont influencer son comportement. Visiteur de passage, il est interpellé par la dimension sociétale de Manger. Il vient au musée pour trouver des réponses à ses questions, pour valider ou invalider son comportement alimentaire. Bien que sensible à la qualité esthétique de la scénographie, il se montre peu attentif aux dispositifs humoristiques en temps que tels, qu'il considère comme un moyen de sensibiliser les plus jeunes aux enjeux d'une thématique. La figure du «visiteur inassouvi ", regroupe des personnes accompagnées par des enfants ou qui n'ont pas prévu plus de deux heures pour voir l'exposition. Elles expriment un sentiment de frustration 
au sortir de la visite. La densité des informations, l'importance des sollicitations sensorielles et/ou la différence des perceptions selon l'âge, les oblige à choisir dans le parcours des points d'arrêt pour en éviter d'autres. Les dispositifs humoristiques servent de ponctuation pour «se détendre » ou, à l'inverse, ne font pas l'objet d'une attention particulière. Cependant, l'exposition qui est vécue comme un objet impossible à englober, trop fournie, voire "fatigante», provoque l'envie de revenir au musée, car elle est scientifiquement appréciée.

Tous les adultes accompagnés d'enfants ne réagissent pas de cette façon. Certains se mettent d'emblée au diapason des plus petits avec une intention éducative. Ces « visiteurs parents-enfants » acceptent de ne regarder que ce qui est susceptible d'intéresser les plus jeunes. Les dispositifs humoristiques sont dans ce cas privilégiés puisqu'ils sont investis d'une valeur ludique, celle-ci étant attribuée, selon le sens commun, à l'univers enfantin. C'est là qu'émerge une question. Nous avons en effet remarqué que la destination des propositions humoristiques dans Manger fait débat auprès des visiteurs. Si certains déclarent que le caractère ludique des vidéos et des chansons se destine aux plus jeunes, d'autres visiteurs constatent qu'elles ne leur sont pas totalement accessibles. Il y aurait par conséquent une différence dans la pratique de ces dispositifs selon que l'on est un adulte ou un enfant. Supports de savoirs pour les uns, les vidéos d'artistes ne seraient qu'une forme attractive pour les plus jeunes. Ce résultat se confirme dans les entretiens avec les 4 à 10 ans qui ne relèvent pas prioritairement ces dispositifs, très appréciés des adultes. Les enfants se focalisent plutôt sur la salle des crottes et sur la table des sons. Les propositions humoristiques de l'exposition toucheraient donc partiellement les plus jeunes et plus largement les adultes. Pourtant la communication du musée est construite en direction des enfants qui sont une cible marketing non négligeable. On remarque précisément que l'affiche de l'exposition représente un ours en peluche. Ce choix créé un horizon d'attente pour une exposition qui ne s'adresse pas précisément aux enfants. L'amusement dans Manger est d'abord un plaisir d'adulte, même si les enfants qui les accompagnent se rendent complices de l'expérience des plus grands, en développant à leur contact, leur propre sens de l'humour. 
En fin d'exposition, la salle des crottes avec la photographie agrandie d'un anus de jument surprend le visiteur.

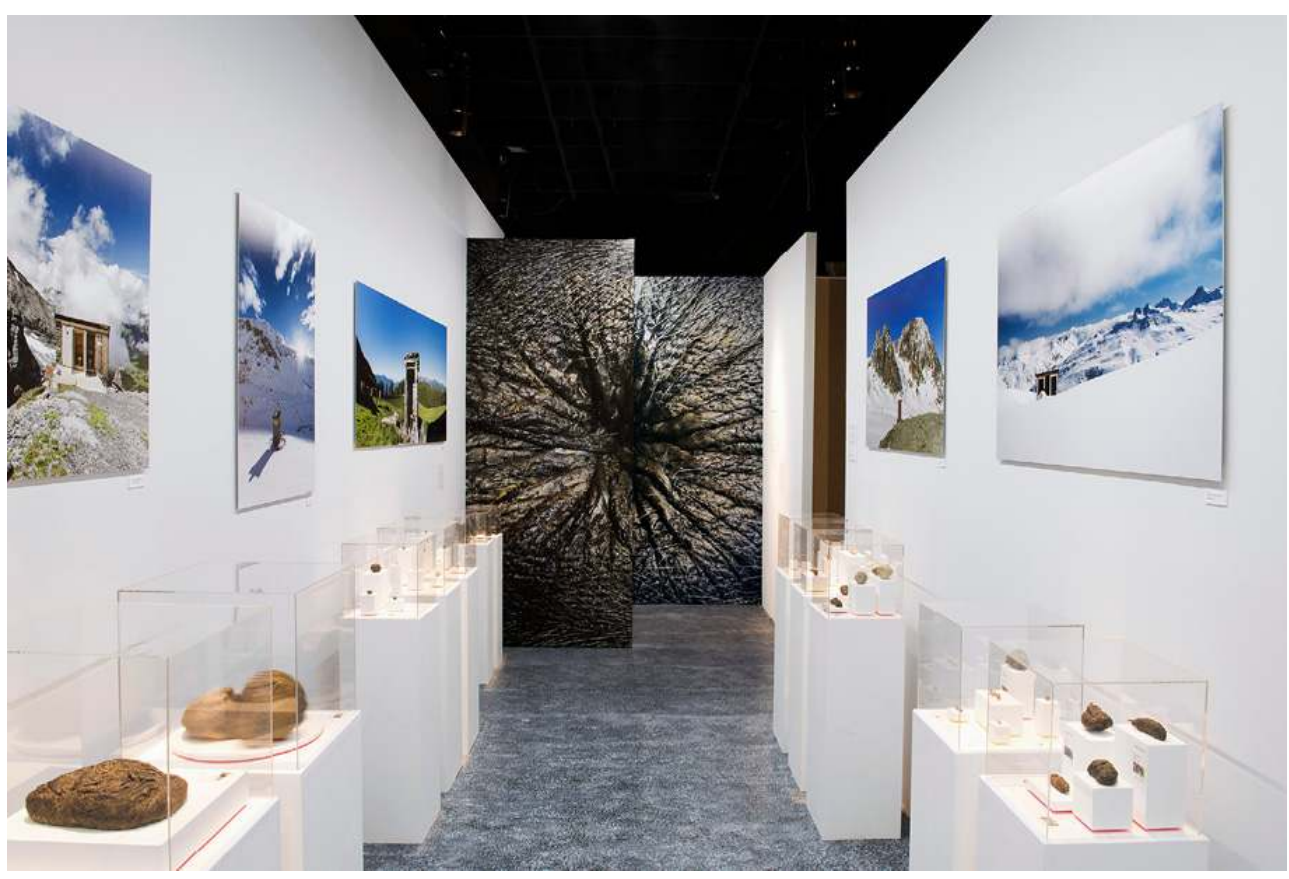

(c) Muséum de Neuchâtel

\section{Conclusion}

Les résultats présentés dans cet article valorisent de manière extrêmement synthétique et donc partielle, la recherche menée au MHN sur l'écriture humoristique de Manger, la mécanique du ventre, une exposition que nous considérons comme représentative d'une aventure collective entre un musée et des artistes sur un sujet scientifique qui est aussi un sujet de société. La matérialisation de cette collaboration musée/artistes dans un projet d'écriture expographique multimédia a convergé vers une scénographie immersive et participative, enrichie de moments drôles, cocasses, parfois truculents qui réalisent un discours singulier, qualifié ici, d'écriture humoristique. Les résultats de notre étude attestent que cette démarche mérite d'être considérée comme un genre de discours muséographique à part entière qui ne se limite pas à favoriser le plaisir du visiteur. Lorsqu'elle est développée comme ici au cœur des contenus, elle stimule, elle interpelle et conduit vers une appréhension sérieuse des connaissances scientifiques. Ainsi l'écriture humoristique contribue à souligner les missions culturelles et pédagogiques essentielles des musées de science contemporains : informer, éduquer, garder l'esprit critique.

Mais tous les musées ne sont pas aptes à mobiliser cette forme muséographique. Il faut avoir cultivé un sens de l'humour et de la dérision vis-à-vis de soi-même (comme professionnel d'un musée), à l'égard des hommes en général et des relations sociales ainsi qu'avec les phénomènes naturels et scientifiques. Il faut aussi pouvoir entretenir une connivence avec ses publics, complicité élaborée sur la durée. L'écriture expographique humoristique nécessite par conséquent une alliance subtile de sérieux et de léger, de rigueur et de dérision, de spontanéité et de ténacité qui loin d'apparaître comme contradictoires, esquissent une nouvelle façon de transmettre la connaissance. Enfin, 
cette recherche de terrain confirme qu'à une époque où les youtubers en médiation scientifique séduisent les publics internautes en les faisant rire, l'exposition de science amusante continue d'assumer sa mission d'éducation à la prise de conscience par le grand public des implications environnementales, sanitaires et sociales du comportement humain dans la nature. Ces résultats et d'autres hypothèses pressenties sur les enjeux de la collaboration musée-artistes dans des expositions humoristiques nous laissent escompter d'autres projets de recherche de ce type à l'avenir dans le champ muséal.

Charaudeau, P. Des catégories pour l'humour? Questions de communication, 2006, $\mathrm{n}^{\circ} 10$, pp. 19-41.

Chaumier, S. Science et humour peuvent-ils faire bon ménage dans une exposition? Bulletin de l'Amcsti, n³6 2012, pp. 3-6.

Ellenbroek, F.-J.-M. Les tabous et l'humour dans les musées, La Lettre de l'Ocim, n54, 1997, pp. 25-27.

Jacquat, M. De l'humour au musée, oser en vaut la peine, La Lettre de l'Ocim, n54, 1997, pp. 28-31.

Poli, M.-S. et Triquet, É. Le kékragône avenionis : un symptôme d'exposition, in Majastre, J.-O. et Pessin, A. Du canular dans l'art et la littérature. Quatrièmes rencontres internationales de sociologie de l'Art de Grenoble. Paris : L'Harmattan, 1999, pp. 219-240.

Poli, M.-S. Éducation et musée, in La muséologie : 20 ans de recherches, Culture \& Musées, numéro Hors-série, Acte Sud, 2013, pp. $165-187$.

Présentations de l'exposition sur internet: www.youtube.com/watch?v=qpZagqQ_Opc www.youtube.com/watch?v=aN3x2d8grUU

\section{NOTES}

1. Crad' expo (2017) et Zizi sexuel l'expo (2014-2015), Cité des Sciences et de l'Industrie ; Bêtes de sexe la séduction animale (2012-2013), Palais de la découverte ;

Au fil des araignées (2014, musée de Grenoble) ; Confidences d'Outre-tombe (2015), musée Dauphinois...

2. MHN : www.museum-neuchatel.ch/index.php/approfondir/les-expositions/les-anciennesexpositions.

3. Exposition désormais nommée en forme raccourcie Manger dans l'article.

4. Cet article doit beaucoup aux enquêtes réalisées par Ambre Mendoza durant son stage au MHN, dans le cadre de sa première année de Master « Médiation de la culture et des patrimoines » de l'université d'Avignon et des Pays de Vaucluse.

5. L'équipe de conception est composée de Christophe Dufour, directeur ; Célia Bueno, conservatrice et directrice adjointe ; Jessica Littman, conservatrice adjointe des invertébrés ; Pauline Demontmollin, chargée de relation publique, de la conservation des archives et de la conception d'expositions ; Thierry Malvesy, conservateur en sciences de la terre ; Anne Remseyer, scénographe et designer. 
6. Poli, M.-S. et Triquet, É. Écrire au muséum : pour une approche résolument transdisciplinaire, Lidil, n²3, 2001, pp. 45-68.

7. Jacobi, D. Discours d'exposition et point de vue, in Les musées sont-ils condamnés à séduire? Et autres écrits muséologiques, Les Essais médiatiques, 2017, p. 152.

8. Ce groupe au physique de hypsters se revendique volontiers de Georges Brassens, des Frères Jacques ou de Boby Lapointe.

9. Notre panel regroupe 25 adultes âgés de trente à soixante-dix ans, 3 jeunes adultes et 6 enfants de quatre à douze ans.

\section{RÉSUMÉS}

Cette étude de réception de l'exposition Manger, la mécanique du ventre - présentée en 2016-2017 au muséum d'Histoire naturelle de Neuchâtel - permet de mesurer les effets de l'humour sur la compréhension par le public du contenu scientifique. Elle montre également que l'écriture humoristique est un genre de discours muséographique qui contribue à affirmer les missions culturelles et pédagogiques des musées de science.

\section{INDEX}

Mots-clés : marie-sylvie.poli@univ-avignon.fr

\section{AUTEURS}

\section{MARIE-SYLVIE POLI}

Professeur émérite à l'université d'Avignon et des

Pays de Vaucluse, Centre Norbert Elias, équipe Culture et Communication 